\title{
A UK financial conditions index using targeted data reduction: forecasting and structural identification
}

\author{
George Kapetanios * \\ Kings College, University of London \\ Simon Price \\ Garry Young \\ Essex Business School, City University and CAMA NIESR and CFM
}

November 29, 2017

\begin{abstract}
A financial conditions index (FCI) is designed to summarise the state of financial markets. Two are constructed with UK data. The first is the first principal component of a set of financial indicators. The second comes from a new approach taking information from a large set of macroeconomic variables weighted by the joint covariance with a subset of the financial indicators (a set of spreads), using multivariate partial least squares, again using the first factor. The resulting FCIs are broadly similar. They both have some forecasting power for monthly GDP in a quasi-real-time recursive evaluation from 2011-2014 and outperform an FCI produced by Goldman Sachs. A second factor, that may be interpreted as a monetary conditions index, adds further forecast power, while third factors have a mixed effect on performance. The FCIs are used to improve identification of credit supply shocks in an SVAR. The main effects relative to an SVAR excluding an FCI of the (adverse) credit shock IRFs are to make the positive impact on inflation more precise and to reveal an increased positive impact on spreads.
\end{abstract}

JEL Codes: C53

Keywords: Forecasting; Financial conditions index; Targeted data reduction; Multivariate partial least squares; Credit shocks

\section{Introduction}

Financial variables have desirable features for policymakers who wish to assess the current state of the economy (nowcast) and forecast the future, and especially so for central bankers. There are several reasons for this. The data are intrinsically forward looking and likely to incorporate

*Correspondence should be addressed to George Kapetanios, george.kapetanios@kcl.ac.uk. 
market expectations about macro data. They may in themselves directly influence the future state of the economy. They are timely and available at high frequencies, so even if ex post they contain no information not available in other data that will eventually be published, they may still be useful in real time when attempting to understand and forecast macroeconomic developments. Moreover, since the onset of the financial crisis in 2007 the monetary transmission mechanism has arguably altered. In the United Kingdom, as in other economies, once policy rates hit the effective lower bound, policy involved asset purchases on a large scale, intended to impact asset yields. While conceivably it used to be the case that a small set of differentlydated interest rates was able to capture relevant financial conditions, that is no longer likely. For example, spreads over Bank and risk-free rates have altered, and credit rationing has been prevalent.

For all these reasons, there has been a renewed interest in financial conditions indices (FCIs). These include for example Angelopoulou et al. (2013) for the EU, Hatzius et al. (2010) for the USA, Gauthier et al. (2004) for Canada and Guichard et al. (2009) and Paries et al. (2014) for variously euro area and some OECD countries, although they were largely developed and published before the onset of the crisis. Regularly produced FCIs include those from Bloomberg, Deutsche Bank, Goldman Sachs, the OECD and the Federal Reserve Bank of Kansas City Financial Stress Index. In this paper we use a hitherto untried method (multivariate partial least squares, MPLS) to construct a new FCI for the UK, as well as a more standard method using principal components (PC).

This innovation combines the insight that large data sets contain a rich set of information about underlying drivers in the economy with the desirable feature of FCIs that they are focussed on or informative about the financial sector. Our approach produces a summary of financial conditions that, uniquely, uses information from a separate large macroeconomic dataset. This is useful in itself as a summary measure, but also turns out to help forecast a variable of interest, namely an interpolated monthly series of GDP, with an advantage over the PC method in a recursive exercise using the longest possible estimation sample. And beyond that we can use the FCI to better identify credit supply shocks in a small structural vector autoregression (SVAR). Using the new FCI makes a material difference to some key impulse responses.

\section{Constructing FCIs}

The history of FCIs is well described elsewhere, so we will be brief. They grew out of an earlier cottage industry that was in the business of producing Monetary Conditions Indices (MCIs). MCIs combine a set of monetary indicators such as money stocks, interest rates and the exchange rate with weights obtained by various means, which are used as summary measures of monetary tightness and to nowcast or forecast. Arbitrary weights may be used, but data-driven methods are generally preferred. A frequently used method, pioneered by the Bank of Canada in the 
1990s, is to weight series using the correlation with or regression coefficients in an equation explaining a variable of interest (typically inflation), on the grounds that this increases the usefulness of the indicator in this context. In a similar spirit, some recent FCIs (e.g. Swiston (2010)) have been constructed using VARs. One reason why the regression based MCIs fell out of fashion at some central banks was the difficulty in interpreting them, a point made forcefully in Ericsson et al. (1998), who point out that MCIs are not underpinned by a structural model derived from stable underlying microeconomic foundations (a critique that carries over to FCIs). As such, their stability and predictive power is questionable. They are certainly vulnerable to the Lucas critique: policy changes (or, more precisely, policy regime changes) reduce their utility. The issue is essentially one of identification.

Structural models, by contrast, allow us to understand the primitive shocks driving all aspects of the economy, including the monetary and financial sectors. In principle it would be possible to estimate such models, identify financial shocks and construct impulse responses that describe the dynamics of those shocks as they are transmitted through the economy. However, structural (DSGE) models with a role for a credit sector and for unconventional monetary policy are only now beginning to be explored, with a variety of financial frictions included and as yet no candidate for the canonical model. Moreover, many of the frictions that have been introduced have small effects on the monetary transmission mechanism. And in the standard New Keynesian model, central bank balance sheet operations have no effect due to a neutrality result discussed in Curdia and Woodford (2011), and the additional mechanisms used there that make asset purchases useful allow no role for government securities. Features such as market segmentation, preferred habitats, costs of adjusting portfolios or heterogeneity among different agents are needed, and DSGE models including them are in their infancy (e.g. Andrés et al. (2004), Gertler and Kiyotaki (2010)) and Harrison (2012)). So although it would be desirable to have identifiable structural shocks, at present structural models are insufficiently well developed to deliver them.

An alternative might be to use an SVAR using some looser theoretical structure. For example, Swiston (2010) estimates an SVAR and constructs impulse responses from the estimated financial shocks, and examines the cumulated impact on GDP growth. But the approach inevitably pivots around the question of how to identify the shock of interest, which as the discussion above indicates, remains uncertain. Swiston (2010) employs a Cholesky decomposition, which may be appropriate in some cases (e.g. identifying a monetary shock in a simple VAR with interest rates and, say, growth and inflation) but in general is arbitrary. An alternative approach is to use sign restrictions to identify shocks as is done in Barnett and Thomas (2013), as the restrictions are rooted in theory but do not require a tight specification. The problem then becomes one of timeliness, as typically models are quarterly. Furthermore results are inevitably subject to model and parameter uncertainty, which equally applies to estimated DSGE models. Despite these reservations we identify credit supply shocks using sign restrictions below, but to check 
the usefulness of our approach rather than to generate an FCI.

Nevertheless, FCIs may be useful as descriptive statistics and for forecasting. And from a practical point of view reduced-form statistical techniques may be the only means available to assess the impact of financial shocks and unconventional policy instruments (which work through financial and credit markets). Thus we take it as given it is worthwhile constructing such indices.

As with MCIs, some commonly reported FCIs simply average variables to provide an atheoretical summary measure. Regression techniques are not widely used. Recent data-driven methods use principal components to extract common factors from a group of financial variables, which is then interpreted as an FCI. Methods also allow time variation in the weights and the relationship with the macroeconomic variables of interest (e.g. Koop and Korobilis (2013)). It should be clear that we too accept that reduced form techniques like these are useful. But there is an alternative that has not yet been explored. Namely, aggregating indicators via multivariate partial least squares.

In modern macroeconomic models there are assumed to be a small number of structural shocks driving the economy. As a general proposition, we may prefer to use as much data as possible to extract these shocks, recognising that shrinkage of some form may be required to do so. Empirically, it is well established that macroeconomic variables jointly co-vary and that a small number of orthogonal factors do indeed explain a large proportion of the joint variance of any data set. This is, of course, part of the attraction of factor extraction by principal components. The approach is intrinsically reduced form, using statistical criteria to identify the factors, so common factors are not structurally identified with economically meaningful interpretations. Any principal component will be a combination of primitive shocks. In the context of financial indicators, this seems to rule out the use of a large 'data rich' dataset including a range of macroeconomic data, and practical attention has been restricted to sets of variables that may be described as 'financial', in the hope that there is a unique 'financial' factor driving them. But this may not apply. As with all economic data, financial variables move because they are subjected to all primitive shocks. A well-defined structural model would enable identification, but as we have argued we do not have robust models available to enable us to do so. Moreover, structural models typically use a small set of data and consequently do not exploit all the information available to us. Under these circumstances, the decision that has been implicitly made by e.g. Hatzius et al. (2010) or Paries et al. (2014) is that the best that might be done is to look for a common factor among the financial variables (transformed to stationarity), ignoring other information, and refer to it as an FCI. But even then there remains ambiguity about what constitutes a financial variable. Both sets of authors include money stocks and the exchange rate, for example, usually included in MCIs, and perhaps better thought of as monetary indicators.

We advocate a halfway house where we extract common factors from a large set $\left(x_{t}\right)$ of 
disparate macroeconomic variables, identifying them by their usefulness in explaining the joint variation between that set and another consisting of financial data $\left(y_{t}\right)$. This remains reduced form - the common factors are not structural in the sense of being derived using an economic model - but quasi-structural in the sense that we are identifying that combination of structural shocks that is important for a subset of data, namely financial. So we focus on a small number of financial variables, but in relation to a much wider macroeconomic data set. In the process we may potentially increase explanatory power by using a large data set (thus better extracting the primitive shocks) without losing financial focus.

If the $y_{t}$ were a single variable there are many ways to do this. Assuming $x_{t}$ is too large for standard regression analysis, then possibilities include Shrinkage Regression, Partial Least Squares (PLS) and Lasso type approaches: see Groen and Kapetanios (2013). In our problem, however, $y_{t}$ is a vector. So specifically, we extract common factors from a large macroeconomic data set, choosing weights by maximising the covariance between those data and a small set of financial indicators, using multivariate partial least squares. It is akin to principal components, which ranks orthogonal transformations of a data set by variance. From an economic point of view, this is arbitrary and the results may not correspond to any interpretable factor. PLS selects factors by additionally using information from the set of (financial) variables of interest. This is also arbitrary in some sense, but may be more interpretable. We can characterise it as part of a class of methods that involve targeted data reduction, in this case reduction of a macroeconomic data set targeted on a set of financial conditions indicators. And, to be clear, unlike a PC, it is the factor constructed by combining macroeconomic data we use, not a combination of financial variables. We use FCI-M6 to denote the new index, which we explain more fully in the following sections.

\section{$3 \quad$ Multivariate partial least squares}

For reference, it is helpful to begin with a simple framework for studying data-rich based modeling methods provided by the general equation

$$
y_{t}=\alpha^{\prime} x_{t}+\epsilon_{t} ; \quad t=1, \cdots, T
$$

where $y_{t}$ is the target of the exercise and $x_{t}=\left(x_{1 t} \cdots x_{N t}\right)^{\prime}$ is an $N \times 1$ vector of explanatory or indicative variables. It is assumed (as is often the case in macroeconomic applications) that the number $N$ of indicator variables is too large for $\alpha$ (also $N \times 1$ ) to be determined by standard methods such as ordinary least squares.

In comparison with some other approaches, partial least squares is a relatively recent addition to regression methods. It was introduced to facilitate the estimation of multiple regressions when there is a large, but finite, amount of regressors. Principal components regression as used by, 
e.g., Stock and Watson (2002) has its roots in Pearson (1901). By contrast, Herman Wold began developing techniques in the 1960s leading to the introduction of his NIPALS (Nonlinear Iterative Partial Least Squares) algorithm in 1973 in the context of calculating principal components. He subsequently applied it to PLS itself in 1977: see e.g. Wold (1982). Since then, PLS has received attention in a variety of disciplines, especially in chemometrics, but rarely in economics. de Jong (1993) is a useful introduction. The essential idea is similar to principal component analysis in its use as regressors of factors or components which are linear combinations of the original regression variables, instead of the original variables individually. The major difference is that whereas in PC regressions the factors are constructed taking into account only the values of the $x_{t}$ predictor variables, in PLS the relationship between $y_{t}$ and $x_{t}$ is considered in constructing the factors. There is a limited literature that has considered PLS regression for data sets with a very large number of series, i.e., when $N$ is assumed in the limit to converge to infinity, which is an assumption that has motivated the use of $\mathrm{PC}$ regression for macroeconomic forecasting.

Most of the attention in the PLS regression literature has focused on the univariate case where $y_{t}$ is scalar. We maintain this to explain the basic features and then extend to a vector $y_{t}$. There are a variety of definitions for PLS and accompanying specific algorithms that inevitably have much in common. A conceptually powerful way of defining PLS is to note that the factors are those linear combinations of $x_{t}$, denoted by $\Upsilon x_{t}$, that maximise covariance between $y_{t}$ and $\Upsilon x_{t}$ while being orthogonal to each other. In analogy to PC factors, an identification assumption is needed, in the usual form of a normalisation.

A detailed discussion of a simple algorithm to construct $k$ PLS factors is in Helland (1990). Assuming for simplicity that $y_{t}$ has been demeaned and $x_{t}$ have been normalised to have zero mean and unit variance, a simplified version of the algorithm is given below

\section{Algorithm 1}

1. Set $u_{t}=y_{t}$ and $v_{i, t}=x_{i, t}, i=1, \ldots N$. Set $j=1$.

2. Determine the $N \times 1$ vector of indicator variable weights or loadings $w_{j}=\left(w_{1 j} \cdots w_{N j}\right)^{\prime}$ by computing individual covariances: $w_{i j}=\operatorname{Cov}\left(u_{t}, v_{i t}\right), i=1, \ldots, N$. Construct the $j$-th PLS factor by taking the linear combination given by $w_{j}^{\prime} v_{t}$ and denote this factor by $f_{j, t}$.

3. Regress $u_{t}$ and $v_{i, t}, i=1, \ldots, N$ on $f_{j, t}$. Denote the residuals of these regressions by $\tilde{u}_{t}$ and $\tilde{v}_{i, t}$ respectively.

4. If $j=k$, where $k$ is the maximum number of factors to be extracted, stop, else set $u_{t}=\tilde{u}_{t}$, $v_{i, t}=\tilde{v}_{i, t} i=1, . ., N$ and $j=j+1$ and go to Step 2 .

This algorithm makes it clear that PLS is tractable for very large data sets as each step is computationally simple. $k$ is unspecified, but may in practice be determined by an information 
criterion as in Bai and $\mathrm{Ng}$ (2002). In our case we examine the first three factors. Once PLS factors are constructed $y_{t}$ can be modeled or used to forecast by regressing $y_{t}$ on $f_{j, t} j=1, \ldots, k$. Helland (1988) and Helland (1990) provide a general description of the problem. Helland (1988) shows that the estimates of the coefficients $\alpha$ in the regression of $y_{t}$ on $x_{t}$, as in (1), obtained implicitly via PLS Algorithm 1 and a regression of $y_{t}$ on $f_{j, t} j=1, \ldots, k$, are equivalent to

$$
\hat{\alpha}_{P L S}=V_{k}\left(V_{k}^{\prime} X^{\prime} X V_{k}\right)^{-1} V_{k}^{\prime} X^{\prime} y
$$

with $V_{k_{1}}=\left(\begin{array}{llll}X^{\prime} y & X^{\prime} X X^{\prime} y & \cdots & \left(X^{\prime} X\right)^{k-1} X^{\prime} y\end{array}\right), X=\left(x_{1}, \cdots, x_{T}\right)^{\prime}$ and $y=\left(y_{1}, \cdots, y_{T}\right)^{\prime}$. Thus (2) suggests that the PLS factors that result from Algorithm 1 span the Krylov subspace generated by $X^{\prime} X$ and $X^{\prime} y$, resulting in valid approximations of the regression between $y_{t}$ and $x_{t}$.

Moving from uni- to multivariate PLS where $y_{t}$ is now a vector involves finding linear combinations of $x_{t}$ whose weights $w$ maximise $w^{\prime} \Sigma_{x y} \Sigma_{x y}^{\prime} w$ where $\Sigma_{x y}$ is the covariance between $x_{t}$ and $y_{t}$. More details on this definition and some limited theoretical results may be found in Chun and Keles (2010). A popular and convenient operationalisation of this concept may be had by defining $\tilde{y}_{t}=w_{y} y_{t}$ where $w_{y}$ is given by the eigenvector associated with the maximum eigenvalue of $\hat{\Sigma}_{x y}^{\prime} \hat{\Sigma}_{x y}$ where $\hat{\Sigma}_{x y}$ is the sample estimate of $\Sigma_{x y}$. Then one can run a PLS regression of $\tilde{y}_{t}$ on $x_{t}$. The first multivariate PLS is then obtained straightforwardly, while the second (and, by extension, further) multivariate PLS factors are obtained by focusing on the residuals of $y_{t}$ (rather than $\tilde{y}_{t}$ ) and $x_{t}$ on the first multivariate PLS factor.

\section{Data}

We assembled a set of 28 UK financial indicators as our $y_{t}$ for January 2004 to June 2014, listed in Table 1. Given data availability changes over time, the sample was chosen to have a moderately large number and long sample of variables. The variables are similar in scope to those US indicators used in Hatzius et al. (2010). And similarly they include variables perhaps better described as monetary rather than financial - for example, interest rates (rather than spreads), asset prices, and monetary aggregates. They are listed in Table 1, arranged by broad category. All variables are transformed to stationarity. In the analysis reported here we construct factors using only the subset of six spreads we describe as 'private sector' (series 7-12) as a relatively pure set of financial indicators, and the complete set of 28 series. We consider our baseline FCI-M6 to be that using the restricted set. This is both parsimonious in the variable selection and a natural choice, as it is often suggested that spreads have predictive power for real activity. For example, Mueller (2009) finds that to be the case for US GDP, where he isolates the credit and macroeconomic components, while finding Treasury spreads have no such power. Similarly, Gilchrist et al. (2009) and Gilchrist and Zakrajsek (2012) find that corporate 


\begin{tabular}{ll}
\hline Gilt yield \\
\hline 1 & 10yr gilt \\
\hline Safe spreads \\
\hline 2 & 3m Tbill - Bank Rate spread \\
3 & 2yr gilt - 3m Tbill spread \\
4 & 10yr gilt - / 3m Tbill spread \\
5 & TED Spread (3m LIBOR - 3m Tbill) \\
6 & 3-month LIBOR/OIS spread \\
\hline Private sector spreads \\
\hline 7 & $£$ Baa corporate - gilts spread (NB: Not just UK issuers) \\
8 & $£$ high yield corporate - Baa corporate spread \\
9 & 75\% LTV variable rate mortgage - Bank Rate spread \\
10 & $£$ 10k personal loan rate - 2-year swap rate spread \\
11 & PNFC variable rate lending rate - 3m LIBOR spread \\
12 & Major UK lenders' CDS premia \\
\hline Asset prices & \\
\hline 13 & $£$ real effective exchange rate \\
14 & FTSE 100 \\
15 & Financials market cap (percent of FTSE 100) \\
16 & Composite UK house price indices \\
17 & $£$ price of gold \\
18 & $£$ price of crude oil relative to 2yr MA \\
\hline Lending & Stock of bank lending (M4L) \\
\hline 19 & $£$ commercial paper Issuance (Relative to 24 Month MA) \\
21 & $£$ bond Issuance (Relative to 24 Month MA) \\
22 & Stock of M0 (notes and coins and reserves) \\
\hline Broad money and debt \\
\hline 23 & Stock of broad money (M4-IOFC) \\
24 & Government bonds outstanding \\
\hline Surveys of credit constraints \\
\hline 26 & Factors likely to limit output: Credit/finance \\
27 & Factors likely to limit capital expenditure: External finance \\
28 & Factors likely to limit capital expenditure: Cost of finance \\
\hline
\end{tabular}

Sample 2004m01 to 2014m06; all variables transformed to stationarity

Table 1: $y$ - potential financial indicators 
bond spreads explain a large proportion of the variance of economic activity and in general have predictive power for a range of business cycle activity indicators. Barnett and Thomas (2013) find corporate spreads to be important in understanding UK lending and activity. Moreover, as we see below, it has good forecast performance at low horizons and is generally superior on this metric to other measures.

For the $x_{t}$, we construct a large monthly macroeconomic data set $(\mathrm{N}=82)$ over the same sample, transformed to stationarity. They may be divided into broad categories: a short rate; CPI indices; surveys of activity and expectations; labour market activity; surveys of confidence; house prices; indices of Production; and retail sales. The full list is in Tables A and B in the data appendix. There is a ragged edge (missing data at the end of the sample) for some series. We handle this with random-walk forecasts for the missing observations. In the forecasting exercise reported below we take a quasi-real-time approach, meaning that while we do not allow for data revisions, we do treat the ragged edge consistently, using only data observations that would have been available at any point in time. We fill the missing observations with random walk (no-change) forecasts. In practice, the ragged edge makes next to no difference to the estimated FCI-M6 in the sense that if we truncate the sample to keep the panel balanced, results are very similar.

\section{$5 \quad$ FCIs}

We examine four variants of our FCIs. Specifically, we use PC and MPLS to extract factors from our two sets of $y_{t}$ variables We restrict attention to the first 3 factors in each of the four cases. In the FCI-PC28 case the first factor explains around $34 \%$ of the variance, and the second and third around $14 \%$ and $7 \%$ respectively. We refer to the PC factors from the smaller (6) and complete (28) sets as FCI-PC6 and FCI-PC28 respectively, and similarly the MPLS factors are denominated as FCI-M6 and FCI-M28. In Section 6 we clearly establish that for forecasting purposes FCI-M6 dominates FCI-M28 while FCI-PC28 outperforms FCI-PC6. For brevity we therefore restrict attention to these two best performers, namely FCI-M6 and FCI-M28.

For the principal components (FCI-PC28) we broadly follow Hatzius et al. (2010) and Paries et al. (2014) using the full set of 28 indicators. An innovation in Hatzius et al. (2010) is that they allow for an unbalanced panel, but we restrict attention to a balanced panel, subject to the ragged edge. We diverge from the methodology in these papers in one respect. They both 'purge' the data by using the residuals from a regression of the financial indicator on a set of variables related to 'macroeconomic conditions', such as inflation, a short rate and output growth (and in Paries et al. (2014) also lagged dependent variables). In line with our discussion above on the ubiquity of underlying shocks, we do not see the rationale for this reduced form filter being applied. Hatzius et al. (2010) argue that an FCI should capture exogenous shifts in financial conditions that influence or otherwise predict future economic activity: shocks, in other 
words. Paries et al. (2014) further suggest that these should be serially uncorrelated. We have argued above that while identification of genuine financial shocks is a good aim, it is unlikely that factor extraction methods will deliver them. Instead, we merely aim to produce a summary measure that is useful in the sense of being informative in particular contexts. 'Purging' the data with a prior regression is not appropriate. A simple thought experiment is helpful. If the only common shock in the economy were financial which drives all variables labelled either financial or macroeconomic, then a prior regression would be purging the financial series of precisely what we aim to get information about.

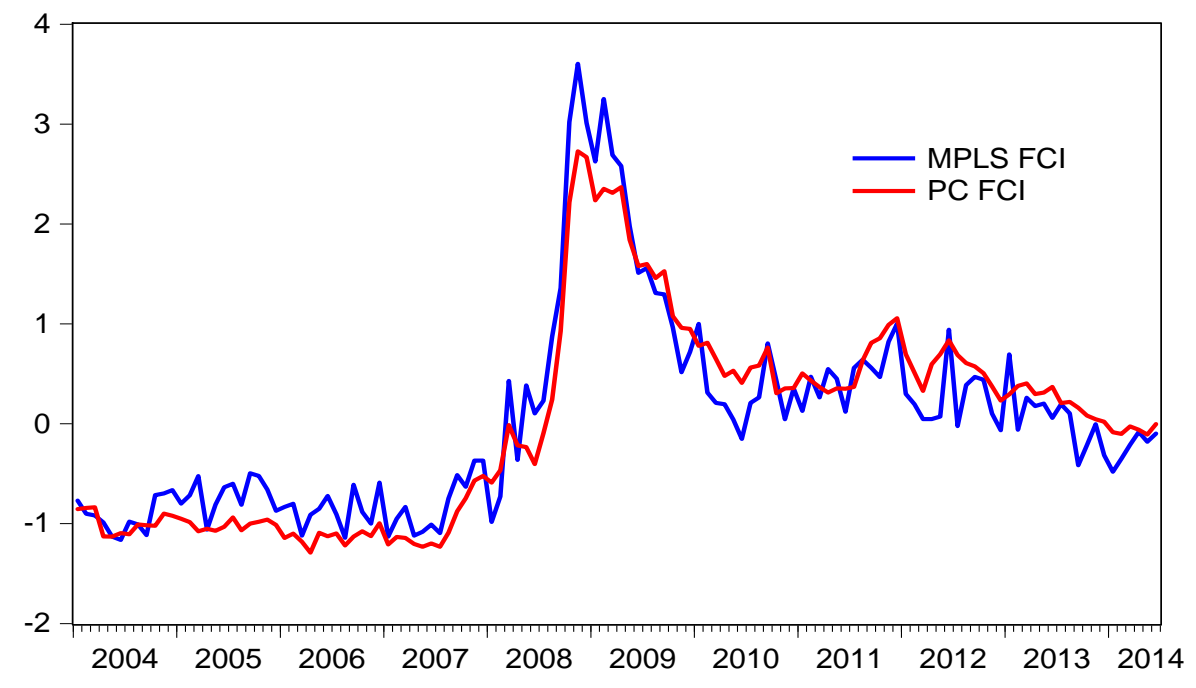

MPLS FCI and PC FCI indicate the MPLS-based FCI-M6 and PC-based FCI-PC28 respectively.

Figure 1: Key UK MPLS and PC financial conditions indices

Figure 1 shows that the FCI-PC28 shows a sharp tightening after the onset of the crisis, followed by a gradual improvement to 2010. The series fluctuated in 2011 but was on a declining trend after early 2012, although it remained above the pre-crisis level at the end of our sample. The new FCI-M6 series based on the six private sector spreads shows a similar profile. The weights on the variables numbered 7 to 12 in Table 1 are $0.31,0.30,0.15,0.15,-0.10$ and 0.18 respectively. There is nothing to constrain them to be positive, although all but one are.

The new MPLS-based FCI is also compared in Figure 2 to the Goldman Sachs FCI (FCI-GS), which does not take account of changes in credit conditions, and is perhaps better interpreted as a monetary index. This vintage was based on 3-month LIBOR rates, 10-year corporate bond rates, the effective exchange rate and UK equity prices with weights of $0.46,0.34,0.17$ and 0.03 respectively. Although the series fell post-crisis before broadly stabilising, it is clear from the pre-crisis period that it is capturing something entirely different, possibly monetary conditions. It may also reflect conditions outside the UK to some degree. 


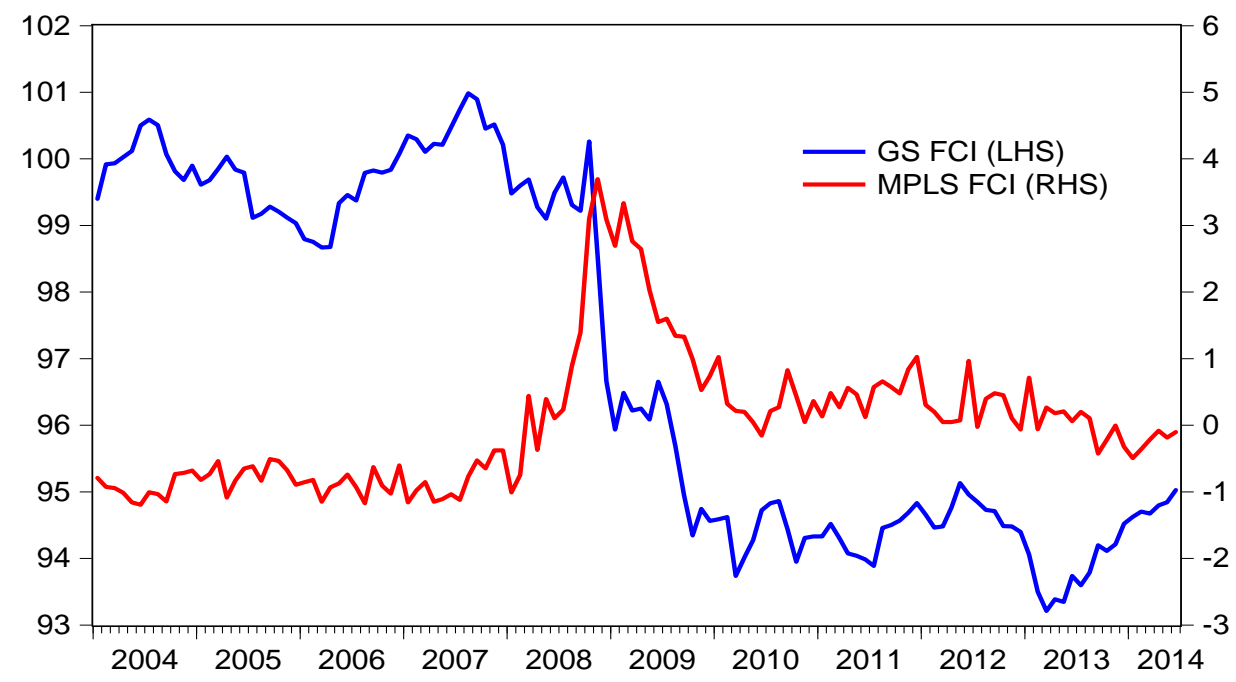

MPLS FCI and GS FCI indicate the MPLS-based and Goldman-Sachs FCIs respectively.

Figure 2: UK MPLS and Goldman Sachs financial conditions indices

It is common to use only the first factor when using FCIs, but with no structural interpretation, it is not obvious why. The second factors from the MPLS (using the narrow set of $y$ ) and $\mathrm{PC}$ are plotted in Figure 3. Once again, they are similar but differ in detail, especially around the crisis. One possible interpretation is that the indices capture monetary conditions - gradually tightening pre-crisis, then loosening as rates fall and the exchange rate depreciates, followed thereafter by a gradual tightening as the real exchange rate unwinds. It is also interesting that the (inverted) second factors move in a similar manner to the Goldman-Sachs FCI (Figure 4). We tentatively interpret the second factor and GS 'FCI' as monetary conditions indices.

\section{Forecast performance}

It is of obvious interest to have timely updates of forecasts, and forecast performance is a useful performance metric. So in this section we recursively estimate the FCIs and forecasting models, and then forecast monthly GDP growth using an $\mathrm{AR}(2)$ augmented with our FCIs. The NIESR series. We use this because it is a timely series (available with a short lag) at a policyuseful frequency (monthly), and also an object of considerable policy interest (being essentially interpolated GDP). It is well established (e.g. Groen et al. (2009)) that good benchmarks for GDP forecasts include low order AR processes, so our benchmark is a simple $\operatorname{AR}(2)$. We also wish to examine the role of different sets of target variables (the $y$ ), the six private-sector spreads and the complete set of 28 financial indicators.

Forecasts are evaluated over the period 2011M06 - 2014M06 (so the evaluation sample is 37 


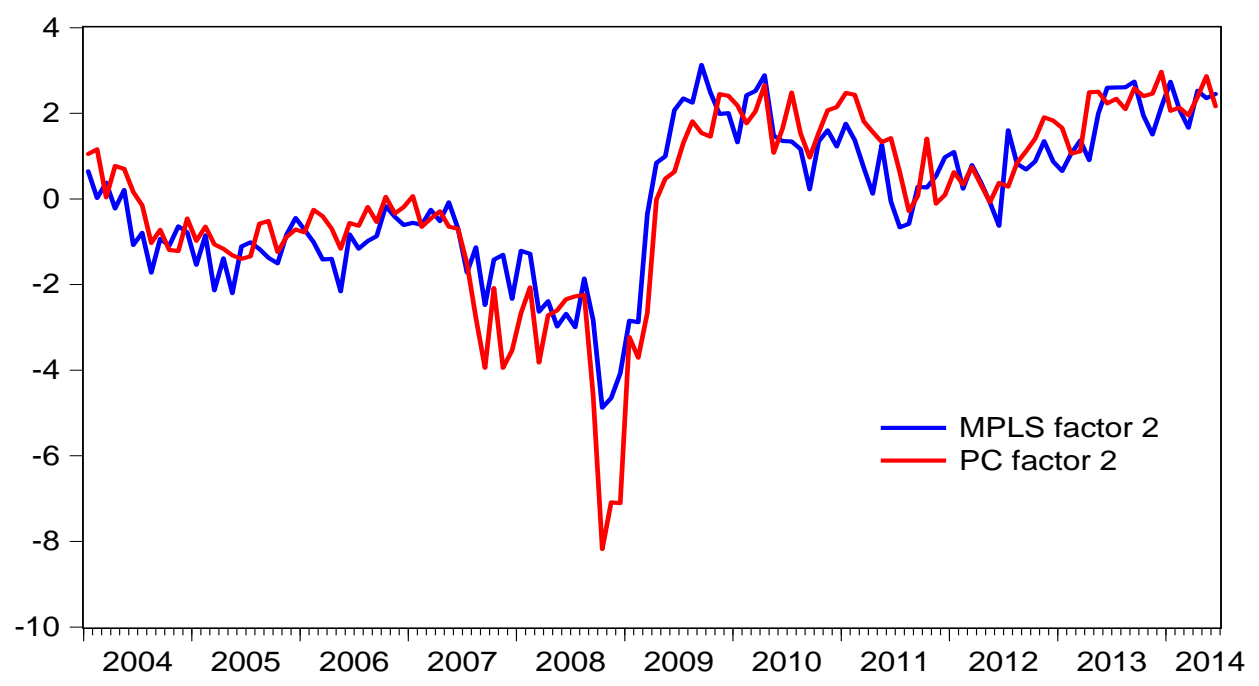

MPLS factor 2 FCI and PC factor 2 indicate the second MPLS and PC factors respectively.

Figure 3: UK MPLS and PC second factors

observations for $h=1$ ) at forecast horizons $h=1$ to 12 months. The data are quasi-real-time, meaning that they take no account of revisions but do include the 'ragged edge'. This does not affect the FCI-GS, as it uses exclusively financial data which are very timely. The tables below show relative root mean square forecast errors (RRMSE) from models using throughout two own-lags and two FCI lags for each of the candidate indices. A value less than 1 indicates better relative performance. In all cases we tested for better performance against the benchmark (and for the benchmark against the model in question) using Diebold Mariano (DM) tests.

Table 2 shows that the FCI-M6 and FCI-PC28 are the two best performers, with the FCIM6 having the best average performance and in 7 out of 12 cases the best performance at any horizon. In four cases the FCI-M6 is significantly better than the benchmark, compared to only one for the FCI-PC28. While FCI-M6 is overall best the FCI-PC28 is not dramatically worse and in some cases better, so we tested each model against the other with A DM test. In no cases were the improvements significant. FCI-M6 is particularly good at the shortest horizon (1 month) with a significant reduction in RMSE of $13.9 \%$, which practically is very important. For MPLS, if the number of target MPLS variables is increased (FCI-M28) then performance deteriorates markedly, while if the PC uses the smaller set of indicators MPC (FCI-PC6) performance falls still more dramatically in most cases, and in one case is significantly worse than the benchmark. The relative performance of small and large $y$ differ between the two methods. With MPLS performance falls off when the target indicators are increased. Specifically, in 9 out of 12 cases performance worsens, in two cases significantly (results not reported in the table), on average by $1.26 \%$, and at horizon 1 by a significant and substantial 14.3\%. By contrast, increasing the PC 


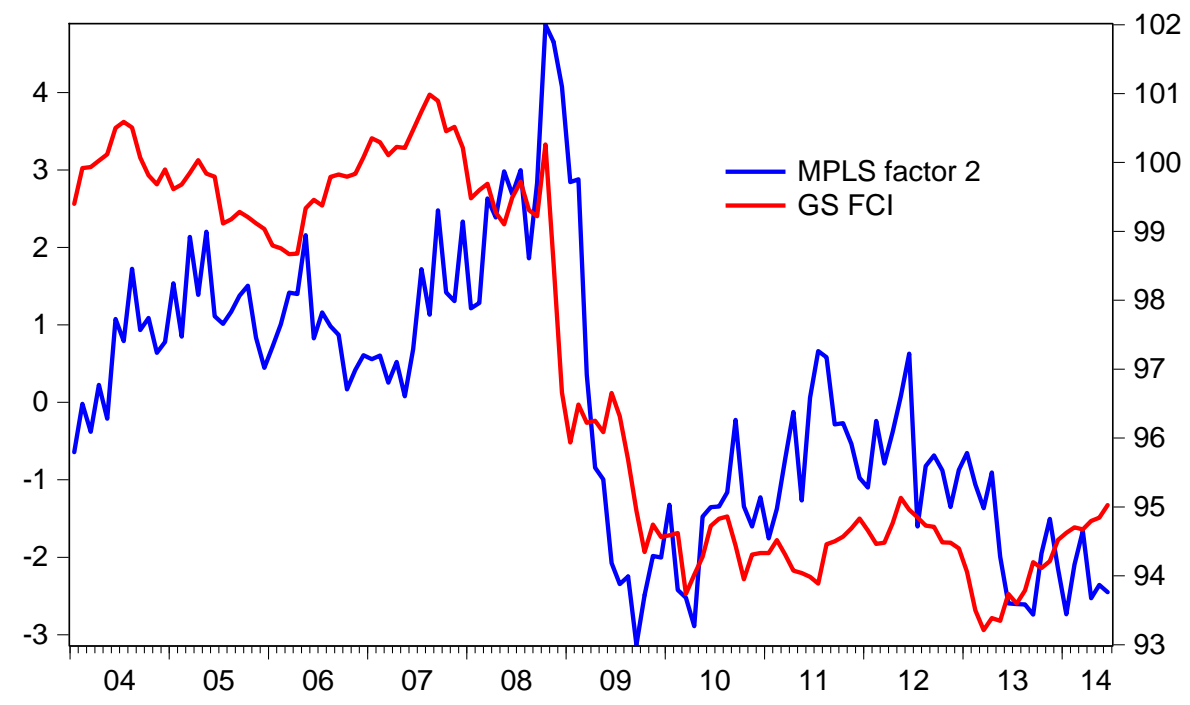

$M P L S$ factor 2 and GS FCI indicate the (inverted) second MPLS factor and the Goldman-Sachs FCI respectively.

Figure 4: UK MPLS second factor and Goldman Sachs financial conditions index

set from 6 to 28 invariably increases performance, on average by $3.8 \%$ and significantly so in half the cases (results not reported in the table). Thus we conclude that while the conventional PC approach is useful, the targeted MPLS method using a small number of spreads almost always dominates, and that in the MPLS using the large target set worsens performance.

\subsection{More factors}

Next we consider whether using more factors improves performance.

Table 3 reports the results of using the first two factors (indicated in the table by, eg, FCI-M6(2) where "(2)" indicates the number of included factors). On average, there is an improvement in performance in each case. The FCI-M28(2) is now on average the best, although at the policy-important first horizon the FCI-M6(2) is ahead by a large margin, with a dramatic and significant RMSE improvement over the benchmark of $20.3 \%$. Moreover, this performance is significantly better than that of the FCI-PC28(2). No other comparisons between the two are significant. Arguably the small FCI-M6(2) results are better than those from the FCI-M28(2), being significantly better than the benchmark in 3 cases, best in half the cases (one more that the for M28) and with the large advantage at the one-month ahead horizon. The PC results are a small improvement over one factor but in almost all cases remain worse than the one-factor MPLS results.

Table 4 shows that adding a further factor marginally worsens average performance in all but the FCI-M28(3), which is marginally improved and is again on average best. Although the 


\begin{tabular}{ccccc}
\hline Horizon & FCI-M6(1) & FCI-PC28(1) & FCI-M28(1) & FCI-PC6(1) \\
\hline 1 & $\mathbf{0 . 8 6 1}$ & $0.864^{*}$ & 0.985 & 0.939 \\
2 & $\mathbf{0 . 9 8 7}$ & 1.015 & 0.995 & 1.030 \\
3 & 0.996 & $\mathbf{0 . 9 6 9}$ & 1.016 & 1.020 \\
4 & $\mathbf{0 . 9 5 7}$ & 0.982 & $0.979^{*}$ & 1.003 \\
5 & $\mathbf{0 . 9 6 8}$ & 0.991 & 1.001 & 1.052 \\
6 & 0.977 & $\mathbf{0 . 9 7 6}$ & 1.003 & 0.995 \\
7 & $\mathbf{0 . 9 6 1 *}$ & 0.993 & 0.994 & 1.028 \\
8 & 0.997 & 0.999 & $\mathbf{0 . 9 9 4}$ & 1.045 \\
9 & $\mathbf{0 . 9 6 1 ^ { * }}$ & 1.006 & $0.989^{*}$ & $1.058+$ \\
10 & 1.051 & $\mathbf{1 . 0 1 9}$ & 1.024 & 1.026 \\
11 & $\mathbf{0 . 9 5 6 ^ { * }}$ & 0.985 & 0.980 & 1.042 \\
12 & 1.002 & 1.033 & $\mathbf{0 . 9 9 8}$ & 1.057 \\
\hline Average & $\mathbf{0 . 9 7 3}$ & 0.986 & 0.997 & 1.025 \\
\hline
\end{tabular}

The table reports relative root mean square error (RRMSE) of an AR(2) augmented with two lags of the first factor from the indicated FCI relative to an AR(2). The best performer in any row is indicated by bold. * records that a DM test indicates the model is significantly better than the $A R$ at $5 \% .+$ records that a DM test indicates the AR is significantly better than the model at $5 \%$.

Table 2: Forecast performance monthly GDP, FCI (first factor) relative to $\mathrm{AR}(2)$

performance of the narrow FCI-M6(3) narrowly deteriorates, there is an interesting pattern. At low horizons (1,2 and 3$)$ they are the best forecasts, there is a further improvement in all horizons up to 6 and the performance at horizon 1 relative to the benchmark is a remarkable $25.0 \%$, which once again is significantly better than for FCI-PC28(3). Again, no other comparisons between the two are significant. At higher horizons performance for FCI-M6(3) generally deteriorates relative to FCI-M6(2), while the large FCI-M28(3) is best at horizons 4 to 9 . With the principal component methods, overall and in the majority of cases performance is worse

The conclusion is that the small-y MPLS based FCI-M6 is a candidate for the best performer and that while using one factor is very effective, using further factors is useful, especially at low horizons. Had we restricted attention to the one-month ahead forecasts, the FCI-M6 would have been unambiguously selected, and by a very large margin. At medium horizons the FCI-M28(3) is a strong contender. The principal components FCI-PC28 also has forecasting power, but less so than for the MPLS variants and with only the first or second factors.

\subsection{Instabilities and lagged $y$}

So using the full available sample for estimation, the FCI-M6 tends to outperform the alternatives we consider in a recursive exercise. But it is possible that there are instabilities in either the factor loadings or the parameters in the forecasting model. On the former, Bates et al. 


\begin{tabular}{ccccc}
\hline Horizon & FCI-M6 (2) & FCI-PC28(2) & FCI-M28(2) & FCI-PC6 $(2)$ \\
\hline 1 & $\mathbf{0 . 7 9 7 ^ { * }}$ & 0.859 & $0.832^{*}$ & 0.947 \\
2 & 0.988 & 1.019 & $\mathbf{0 . 9 5 9}$ & 1.020 \\
3 & 0.971 & 0.973 & $\mathbf{0 . 9 5 2}$ & 1.012 \\
4 & 0.953 & 0.985 & $\mathbf{0 . 9 2 1}$ & 0.980 \\
5 & 0.961 & 0.992 & $\mathbf{0 . 9 5 2}$ & 1.037 \\
6 & $\mathbf{0 . 9 5 1}$ & 0.971 & 0.955 & 0.974 \\
7 & $\mathbf{0 . 9 4 6}$ & 0.987 & 0.961 & 0.985 \\
8 & 0.985 & 0.996 & $\mathbf{0 . 9 4 7}$ & 1.010 \\
9 & $\mathbf{0 . 9 5 1 *}$ & 1.006 & 0.992 & 1.012 \\
10 & 1.066 & 1.008 & 1.023 & $\mathbf{0 . 9 6 8}$ \\
11 & $\mathbf{0 . 9 3 2}$ & 0.973 & 0.974 & 0.983 \\
12 & $\mathbf{0 . 9 9 0}$ & 1.028 & 0.996 & 1.012 \\
\hline Average & 0.958 & 0.983 & $\mathbf{0 . 9 5 5}$ & 0.995
\end{tabular}

The table reports relative root mean square error (RRMSE) of an AR(2) augmented with two lags of the first two factors from the indicated FCI relative to an AR(2). The best performer in any row is indicated by bold. * records that a DM test indicates the model is significantly better than the AR at 5\%. + records that a DM test indicates the AR is significantly better than the model at $5 \%$.

Table 3: Forecast performance monthly GDP, FCI (first two factors) relative to $\operatorname{AR}(2)$

\begin{tabular}{ccccc}
\hline Horizon & FCI-M6(3) & FCI-PC28(3) & FCI-M28(3) & FCI-PC6(3) \\
\hline 1 & $\mathbf{0 . 7 5 0 *}$ & $0.847^{*}$ & $0.836^{*}$ & 0.963 \\
2 & $\mathbf{0 . 9 7 4}$ & 1.015 & 0.989 & 1.016 \\
3 & $\mathbf{0 . 9 4 9}$ & 0.972 & 0.961 & 1.026 \\
4 & 0.930 & 0.985 & $\mathbf{0 . 9 1 7}$ & 0.982 \\
5 & 0.953 & 0.998 & $\mathbf{0 . 9 4 0}$ & 1.051 \\
6 & 0.951 & 0.980 & $\mathbf{0 . 9 4 0}$ & 0.978 \\
7 & 0.957 & 0.995 & $\mathbf{0 . 9 4 7}$ & 0.997 \\
8 & 0.970 & 1.019 & $\mathbf{0 . 9 3 3}$ & 1.009 \\
9 & 1.003 & 1.022 & $\mathbf{0 . 9 8 3 *}$ & 1.017 \\
10 & 1.066 & 1.027 & 1.023 & 0.964 \\
11 & $\mathbf{0 . 9 5 8 *}$ & 0.984 & 0.969 & 0.978 \\
12 & $1.043+$ & $1.053+$ & $\mathbf{0 . 9 9 7}$ & 1.002 \\
\hline Average & 0.959 & 0.991 & $\mathbf{0 . 9 5 3}$ & 0.999 \\
\hline
\end{tabular}

The table reports relative root mean square error (RRMSE) of an AR(2) augmented with two lags of the first three factors from the indicated FCI relative to an AR(2). The best performer in any row is indicated by bold. * records that a DM test indicates the model is significantly better than the AR at 5\%. + records that a DM test indicates the AR is significantly better than the model at $5 \%$.

Table 4: Forecast performance monthly GDP, FCIs (first three factors) relative to $\operatorname{AR}(2)$ 


\begin{tabular}{cc}
\hline Horizon & \\
\hline 1 & $0.852^{*}$ \\
2 & 0.993 \\
3 & 0.990 \\
4 & 0.960 \\
5 & 0.960 \\
6 & 0.982 \\
7 & 0.962 \\
8 & 0.987 \\
9 & $0.955^{*}$ \\
10 & 1.052 \\
11 & 0.954 \\
12 & 0.992 \\
\hline Average & 0.970 \\
\hline
\end{tabular}

The table reports relative root mean square error (RRMSE) of an AR augmented with two lags of the first factor from the FCI-M6 relative to an AR(2) augmented with two lags of the Goldman Sachs FCI $(A R+G S) .{ }^{*}$ records that a DM test indicates the model is significantly better than $A R+G S$ at 5\%. Evaluation period 2011M06 to $2014 M 06$.

Table 5: Forecast performance monthly GDP, FCI-M6 (first factor) relative to FCI-GS

(2013) conclude that full-sample estimation of principal components is remarkably robust to the presence of structural change in the loadings, borne out in both the factor estimation and forecasting dimensions in their empirical investigation in Stock and Watson (2009). Forecasting where there are instabilities was recently surveyed by Rossi (2013). Here a robust strategy is to discount the past in some way, and one simple way to do this is with a rolling estimation window. An obvious issue is how to choose window length. Giraitis et al. (2013a) develop an optimal method for determining this, but the model considered here is too complex for that to be applied. Instead we examine estimation window lengths of 36, 48 and 60 months (compared to the recursive estimation window whose starting sample is 90 months and then extends). Clearly, in the light of the discussion above, there is a tension between allowing forecast model instability and estimation of the factors.

For brevity we restrict attention to the MPLS based FCI with small- $y$ which we have established is a good and robust performer. The results are summarised in Table 6. It emerges that for shorter windows, the FCI-M6 continues on average to beat the AR, and in some cases (window 48) significantly. Similarly, for window length 36 and 60 the FCI-M6 beats the FCI-PC28. This is not true for a 48 period window where in one case $(h=8)$ the FCI-PC28 is significantly better, but overall the results lean towards support for the MPLS.

Given the emphasis in this section on forecasting, we examined the use of lagged ( 1 through 4) financial variables $y$ and the contemporaneous macro data set $x$, speculating that this would result in factors that would be more useful in forecasting (that is, the identified factor may lead 


\begin{tabular}{|c|c|c|c|c|c|c|}
\hline & \multicolumn{6}{|c|}{ Benchmarks } \\
\hline & & $\mathrm{AR}(2)$ & & $\mathrm{AR}$ & )$+\mathrm{FCI}$ & $\mathrm{CC} 28$ \\
\hline \multirow[t]{2}{*}{ Horizon } & \multicolumn{6}{|c|}{ Window length } \\
\hline & 36 & 48 & 60 & 36 & 48 & 60 \\
\hline 1 & 0.948 & 0.845 & 0.903 & 0.970 & 0.981 & 1.005 \\
\hline 2 & 0.998 & $0.973^{*}$ & 0.991 & 0.994 & 0.986 & 0.973 \\
\hline 3 & 0.987 & 0.973 & 0.996 & 0.999 & 1.027 & 1.026 \\
\hline 4 & 0.994 & 0.931 & 0.961 & 0.986 & 1.007 & 0.981 \\
\hline 5 & 0.998 & 0.945 & 0.965 & 0.999 & 1.010 & 0.981 \\
\hline 6 & 1.004 & 0.968 & 0.981 & 0.999 & 1.023 & 1.001 \\
\hline 7 & 0.992 & $0.929^{*}$ & 0.959 & $0.985^{*}$ & 1.005 & 0.972 \\
\hline 8 & 1.014 & 0.954 & 0.988 & 0.999 & $1.036+$ & 1.000 \\
\hline 9 & 0.979 & $0.919 *$ & 0.950 & 0.988 & 0.990 & $0.955^{*}$ \\
\hline 10 & 1.022 & 0.976 & 1.040 & 1.002 & 1.042 & $1.027+$ \\
\hline 11 & 0.987 & $0.908^{*}$ & 0.950 & $0.987^{*}$ & 1.020 & 0.971 \\
\hline 12 & 0.995 & 0.930 & 0.997 & 0.990 & 0.996 & $0.973^{*}$ \\
\hline average & 0.993 & 0.938 & 0.973 & 0.992 & 1.010 & 0.989 \\
\hline
\end{tabular}

The table reports relative root mean square error (RRMSE) of an AR(2) augmented with two lags of the first factor of the FCI-M6 relative to benchmarks. * records that a DM test indicates the model is significantly better than the benchmark at 5\%. + records that a DM test indicates the benchmark is significantly better than the model at 5\%. Evaluation period 2011M06 to 2014M06.

Table 6: Relative forecast performance of an $\mathrm{AR}(2)$ with FCI-M6(1) relative to an $\mathrm{AR}(2)$ and $\mathrm{AR}(2)$ with FCI-PC28 over varying estimation windows 
macro variables). There is a cost to this as we lose effective observations. In the event, forecast performance is little changed. For a lead of 1 , there is an improvement at $h=1$ against the AR benchmark but on average no improvement and no change relative to the PC augmented AR. For a lead of 4 , there is a more marked improvement at $h=1$ against the benchmarks, and on average a small improvement. But there is little forecast advantage gained and it weakens the interpretation as a factor informative about contemporaneous financial shocks, so in the rest of this paper we continue to use that reported above.

\section{$7 \quad$ Identifying credit supply shocks}

We have interpreted our FCI in a quasi-structural sense, but it is also possible to identify credit supply shocks in a genuinely structural sense with identifying restrictions in an SVAR. In this section we therefore use sign restrictions to back out such structural shocks from a small VAR, and ask whether the first factor from FCI-M6, which has the clearest interpretation as an FCI, can help with this identification. We also experimented with additional factors but were unable to identify the SVAR when we did so.

We begin with a VAR including CPI inflation (untransformed), growth (NIESR monthly GDP estimates), loans (PNFC lending, log differenced), bank lending spreads on loans to PNFCs (untransformed) and LIBOR (differenced), which we then augment with the FCI (differenced) from the FCI. The data are shown in Chart 5.

We have 126 monthly observations. We then identify monetary policy and credit supply shocks using the scheme used in Paries et al. (2014) (following e.g. Busch et al. (2010), Gambetti and Musso (2012) and Peersman (2012)).

Specifically, a positively-signed monetary policy shock, i.e. an unexpected increase in the short-term interest rate, depresses activity, prices and loans. A positively-signed credit supply shock associated with a softening of the financing environment raises activity and tightens the policy rate. The impact on prices is left unrestricted as the positive effect on demand can be mitigated by a positive effect on supply. Both a positive monetary policy shock and a negative credit supply shock depress loans and activity. In order to dissociate a positive credit supply shock from an expansionary monetary policy shock, we impose a different sign on the monetary policy rates and impose a fall in the bank lending spread after a positive credit supply shock. In the augmented VAR a negative credit supply shock raises the FCI. We examine one-month responses and following Paries et al. (2014) for the non-financial series (activity, prices and loans) we impose the sign restrictions starting one month after the shock. All this is summarised in Table 7 .

We estimate the impulses using the method employed in Giraitis et al. (2013b).

Figure 6 and 7 show impulse responses following a 1 standard deviation shock up to 40 months for all variables in the SVARs, each estimated with six lags. Figure 6 reports the impulses from 


\begin{tabular}{ccccc}
\hline Variable & \multicolumn{2}{c}{$\begin{array}{c}\text { Adverse credit shock } \\
\text { (rise in FCI) }\end{array}$} & \multicolumn{2}{c}{$\begin{array}{c}\text { Adverse monetary shock } \\
\text { (rise in policy rate) }\end{array}$} \\
\hline & sign & timing & sign & timing \\
\hline Inflation & unrestricted & n/a & - & after one month \\
Growth & - & after one month & - & after one month \\
Lending growth & - & after one month & - & after one month \\
Spread level & + & immediate & unrestricted & $\mathrm{n} / \mathrm{a}$ \\
LIBOR level & - & immediate & + & immediate \\
FCI & + & immediate & unrestricted & $\mathrm{n} / \mathrm{a}$ \\
\hline
\end{tabular}

Table 7: Sign and timing restrictions

an SVAR(5) including inflation, growth, lending growth, spread level and LIBOR level by row, where the first column reports the response to an adverse monetary shock (tightening, higher rates) and the second an adverse credit shock (tightening, associated with higher FCI) together with $90 \%$ confidence bounds generated by bootstrapping the reduced form VAR, its coefficients and reduced form IRFs and using the $A(0)$ matrices obtained by sign restrictions to take the median of the bootstrapped structural IRFs. Figure 7 repeats the exercise for $\operatorname{SVAR}(6)$, with the addition of the FCI-M6.

Our sample is short, and for more than half of the period we are at the effective lower bound, where for obvious reasons it may be hard to identify monetary shocks. The Bank of England uses a 'shadow' interest rate for internal purposes, which takes account of the estimated effect of asset purchases (quantitative easing) and may therefore be negative. But using this in place of LIBOR did not substantially change results. Nevertheless, in most cases they are easily interpretable.

Looking first at the effect of a monetary shock, in the SVAR(5) excluding FCI a monetary shock has a moderately persistent negative impact on inflation which temporarily overshoots by a small amount in the recovery before returning to a persistent negative impact. A strict impact price puzzle is ruled out by assumption, but the negative impact is sustained for roughly nine periods. There is a perverse unconstrained impact effect on output growth, and after the negative restriction kicks in the impulse oscillates around the asymptote, which is less intuitive. Lending contracts briefly. The unrestricted impact on spreads is negative and very persistent. LIBOR reacts positively and the impact decays rapidly and non-monotonically, with a somewhat counter-intuitive trough at 3 months. Including FCI, the inflation profile in the two SVAR(6)S are extremely similar to that in the SVAR(5). The output effect is similar, as is lending growth. The effects on spreads, however, are quite different, initially positive then persistently negative. The effect on LIBOR is broadly similar. Following a negative impact the FCI oscillates between positive and negative values.

A credit shock has a positive and persistent impact on inflation in both the SVAR(5) and 
SVAR(6) but is much larger (and smoother) in the latter case with a small positive impact at long horizons. The implication is that credit shocks have a larger impact on output supply than demand. This raises the question of whether the identified shock is in fact conflating a credit and supply (productivity) shock. Helbling et al. (2011), who identify a credit and productivity shock in a related exercise, argue that it is important to impose a non-negativity (given a positive credit shock) impact on productivity from a credit shock. But we find this hard to justify, given that a part of the impact of credit shocks is likely to be on supply, perhaps via working capital entering the production function as in Fernandez-Corugedo et al. (2011), or research and development expenditure affecting TFP as in Millard and Nicolae (2014). Obvious sign restrictions that would help identify a supply shock are that following an adverse supply shock growth falls and inflation rises, but no other restrictions are evident in our framework. We also note below that our identified credit shock has a large and significant impact in the SVAR(6) case which is hard to understand if the shock were to supply. For the SVAR(5) the impulse response for growth is counter-intuitively initially positive and then oscillating around the asymptote and very small. By contrast, in the SVAR(6) while effects remain small there is initially a negative impact, in accordance with our expectations, before moving to a persistent small positive impact. Lending, where we might expect a clear negative effect, after the initial impact constrained to be negative oscillates around the asymptote in the $\operatorname{SVAR}(5)$ cases. In the SVAR(6) after a counter-intuitive positive spike at 3 months the effect lending is persistently reduced and overall is negative, much more in line with what we expect. Spreads are restricted to initially increase, and in the $\operatorname{SVAR}(5)$ they then decline and become negative. By contrast, in the $\operatorname{SVAR}(6)$ they are uniformly positive, large relative to the effects in the $\operatorname{SVAR}(5)$ and very persistent, which is a more intuitive result. The impacts on LIBOR are similar in the two SVARs. The FCI itself oscillates between positive and negative values briefly ( $\operatorname{SVAR}(6)$ only).

So the addition of our FCI to the SVAR(5) leaves many of the impulses unchanged, but materially and qualitatively affects the estimated effect of a monetary shock on spreads. The positive impact of a credit shock on inflation is much larger and without the counterintuitive impact on growth, although effects remain small. The negative persistent effect on lending is much more intuitive, as is the large uplift in spreads. 

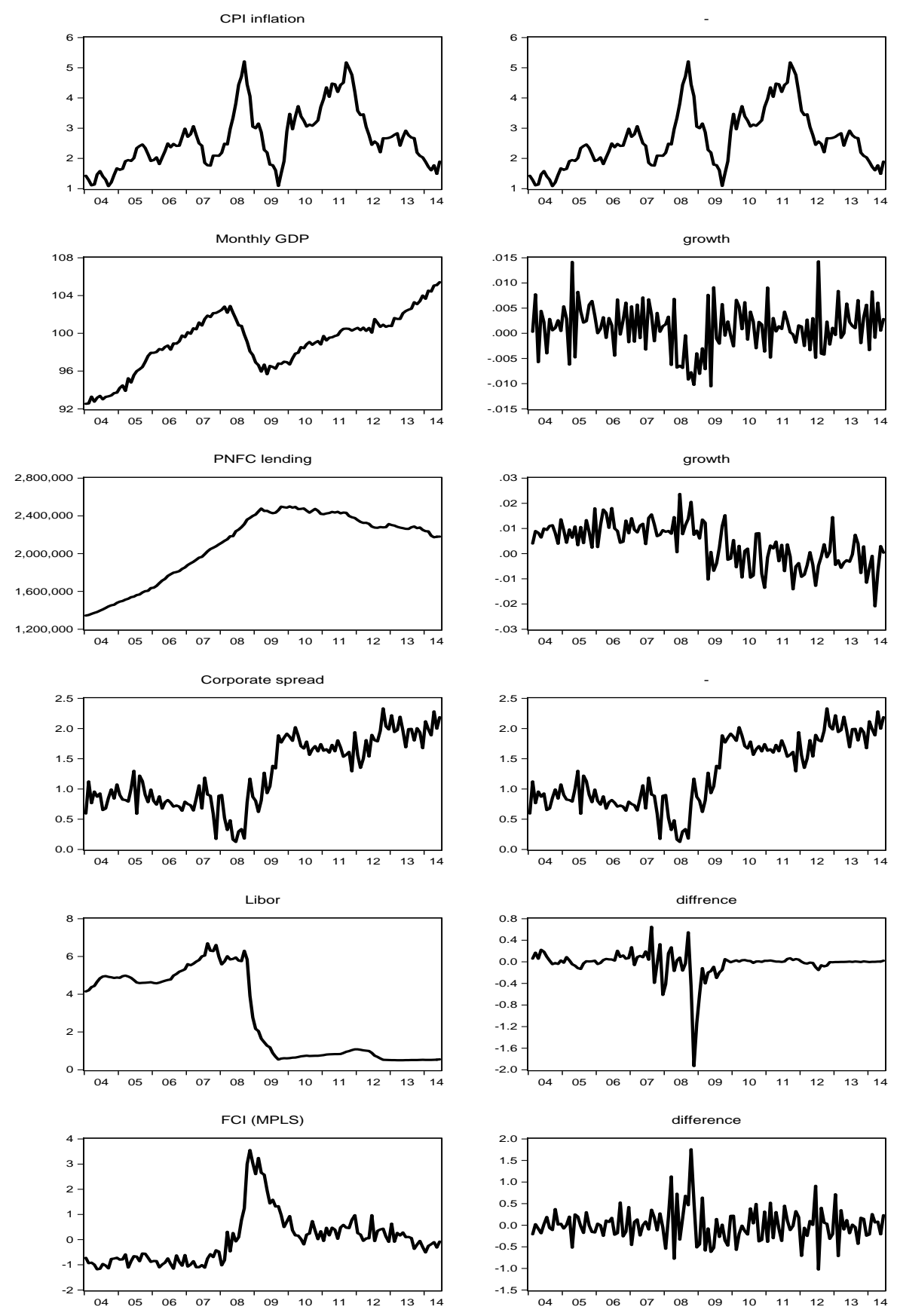

Figure 5: Original and transformed data used in the VARs 
Monetary Shock

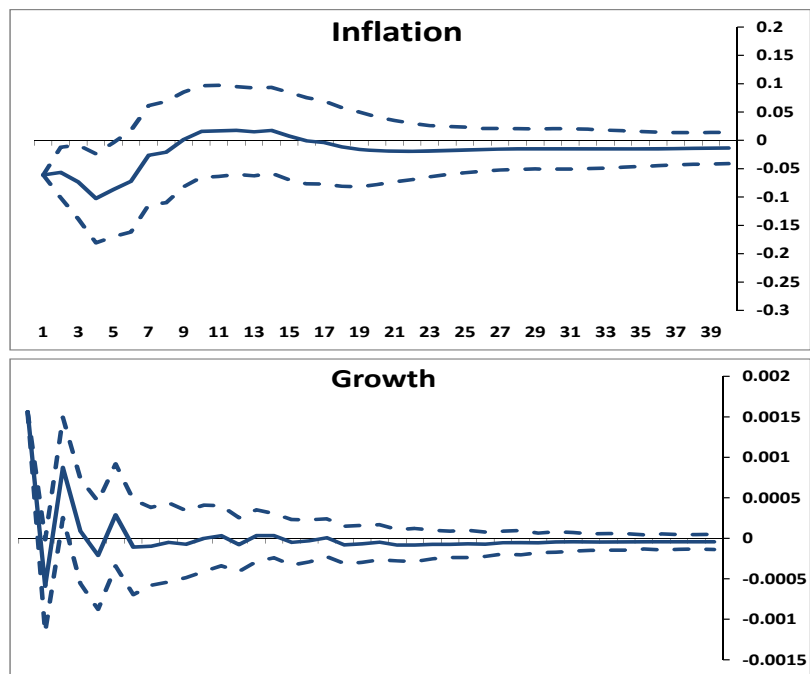

$\begin{array}{llllllllllllllllllll}1 & 3 & 5 & 7 & 9 & 11 & 13 & 15 & 17 & 19 & 21 & 23 & 25 & 27 & 29 & 31 & 33 & 35 & 37 & 39\end{array}$

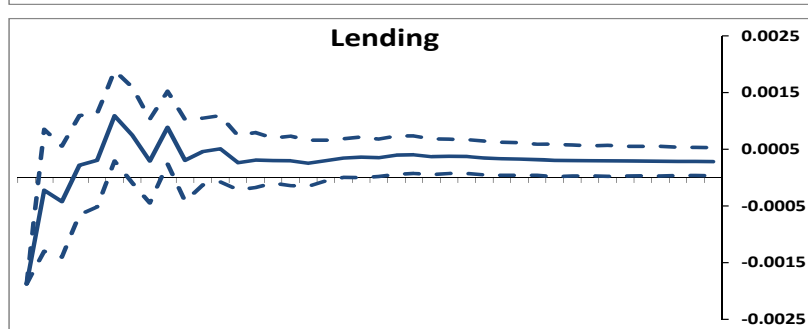

$\begin{array}{llllllllllllllllllll}1 & 3 & 5 & 7 & 9 & 11 & 13 & 15 & 17 & 19 & 21 & 23 & 25 & 27 & 29 & 31 & 33 & 35 & 37 & 39\end{array}$
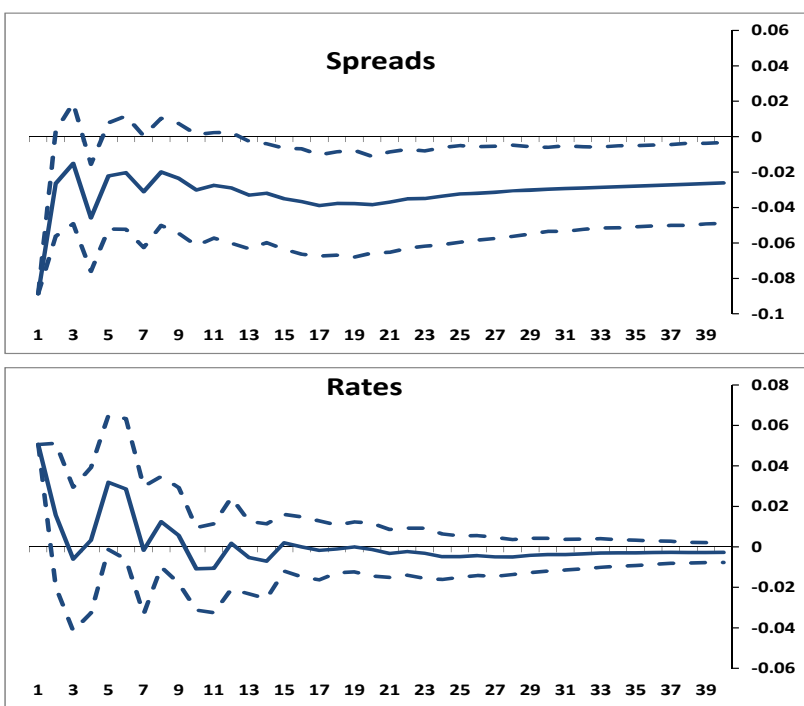

\section{Credit Shock}

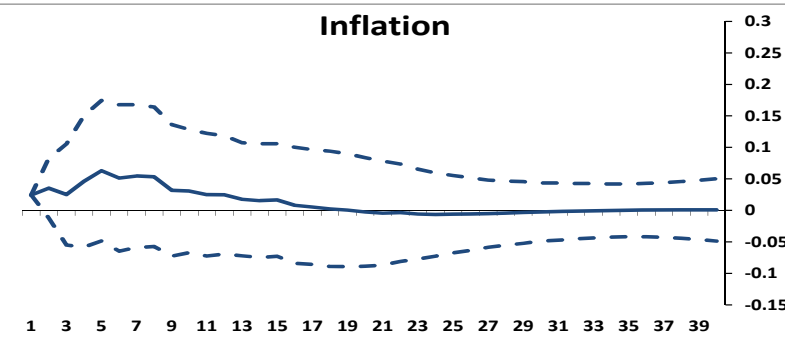

$\begin{array}{lllllll}1 & 3 & 5 & 7 & 9 & 11 & 13\end{array}$

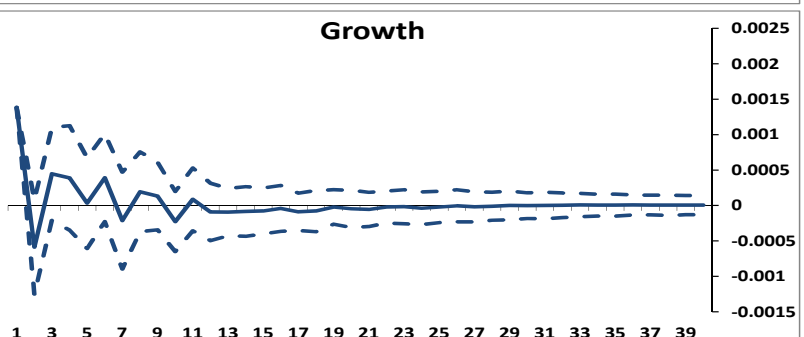

$\begin{array}{llllllllllllllllllll}1 & 3 & 5 & 7 & 9 & 11 & 13 & 15 & 17 & 19 & 21 & 23 & 25 & 27 & 29 & 31 & 33 & 35 & 37 & 39\end{array}$
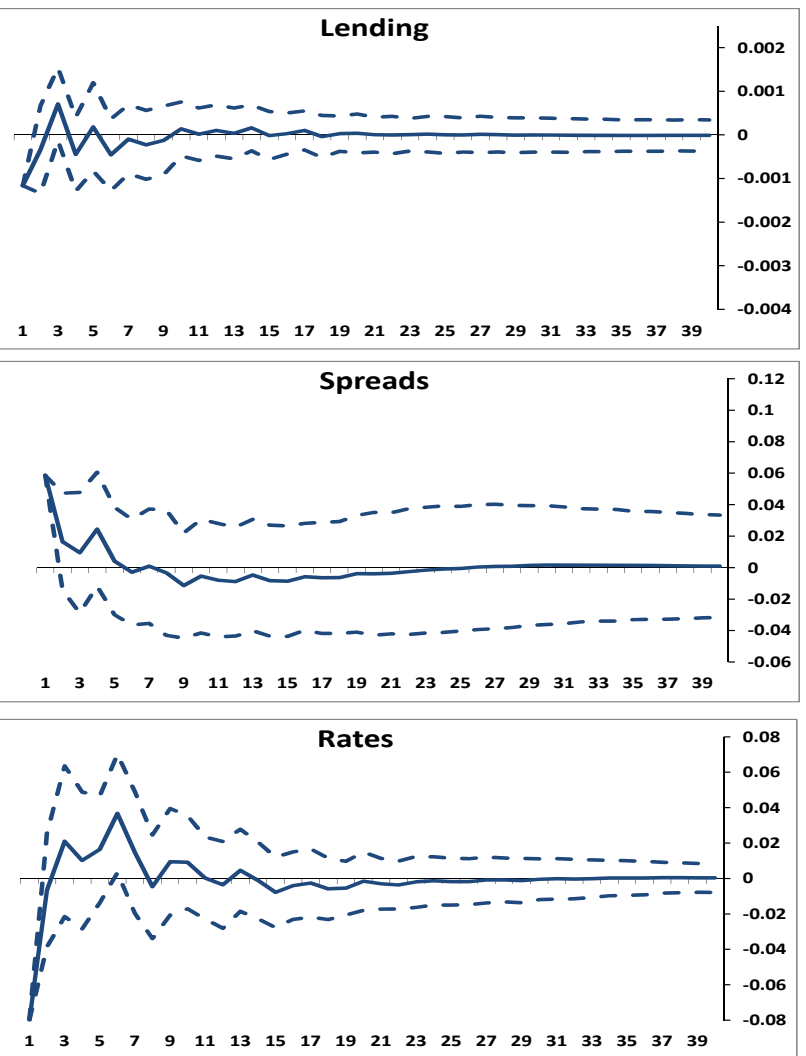

Figure 6: Impulse responses and 90\% confidence bounds following adverse monetary and credit 1 SD shocks, excluding FCI. 

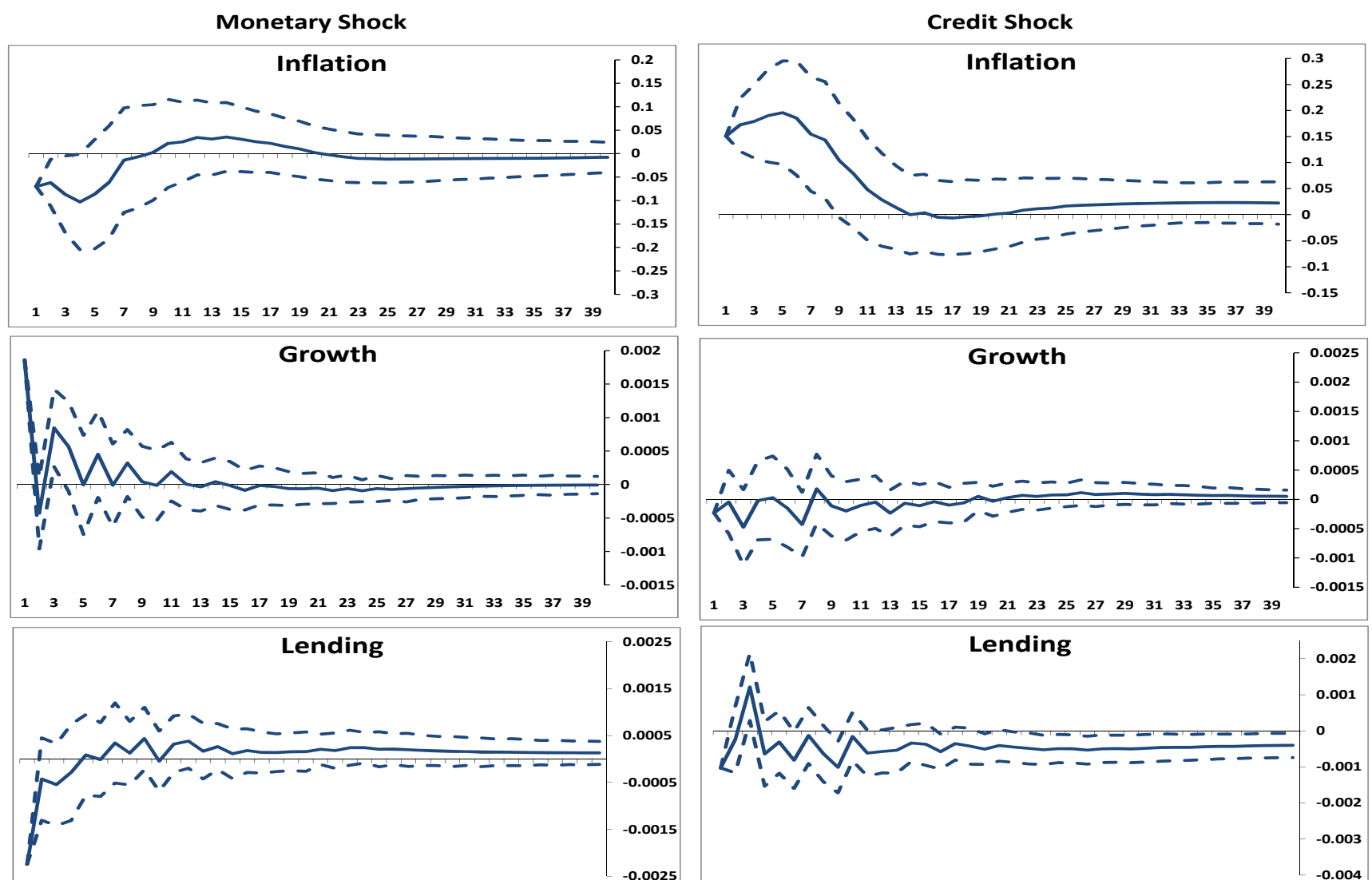

0.0025 1
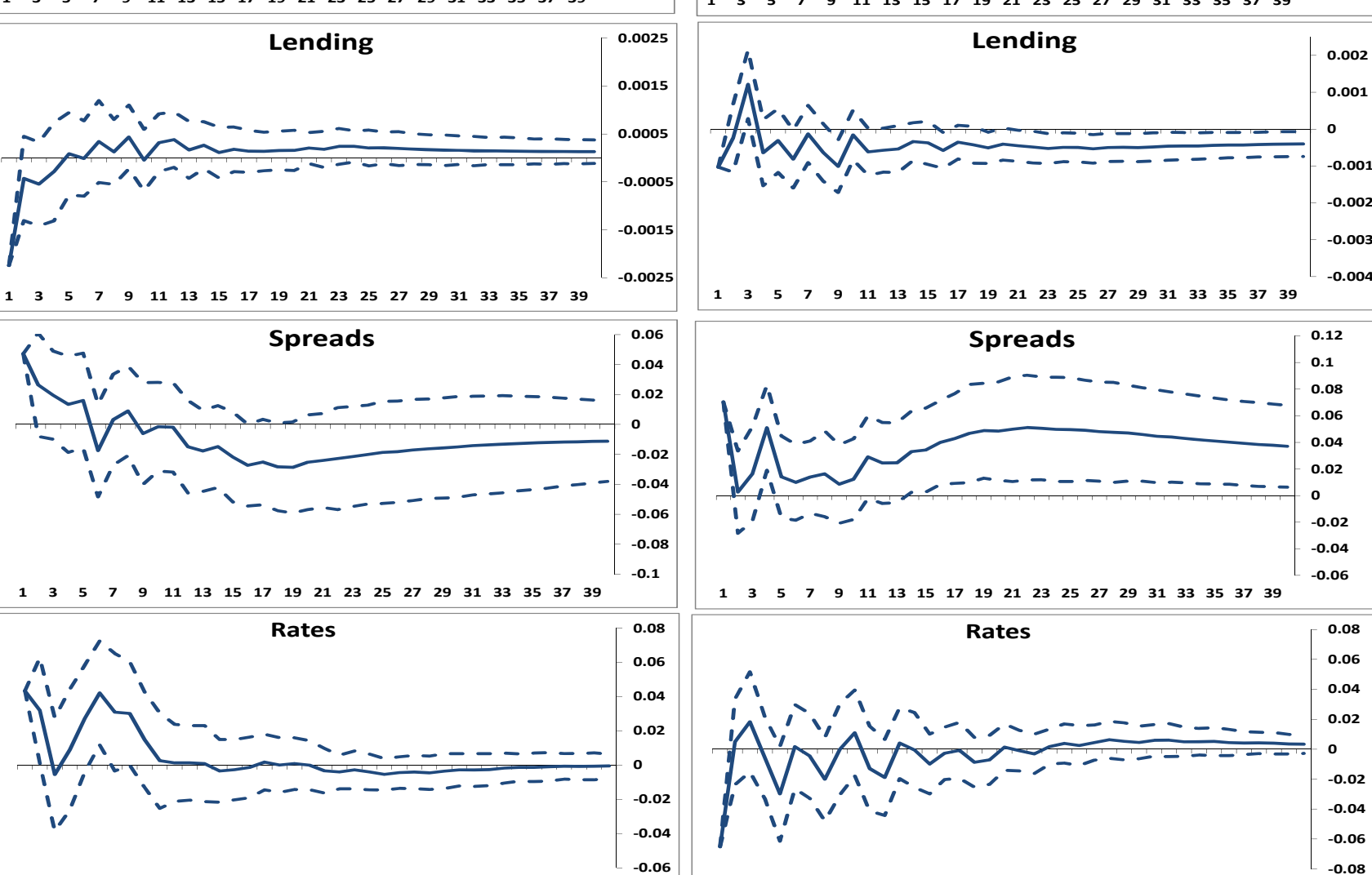

$\begin{array}{llllllllllllllllllll}1 & 3 & 5 & 7 & 9 & 11 & 13 & 15 & 17 & 19 & 21 & 23 & 25 & 27 & 29 & 31 & 33 & 35 & 37 & 39\end{array}$

\begin{tabular}{l}
0.25 \\
0.2 \\
0.15 \\
0.1 \\
0.05 \\
0 \\
-0.05 \\
-0.1 \\
-0.15 \\
-0.2 \\
-0.25 \\
\hline
\end{tabular}

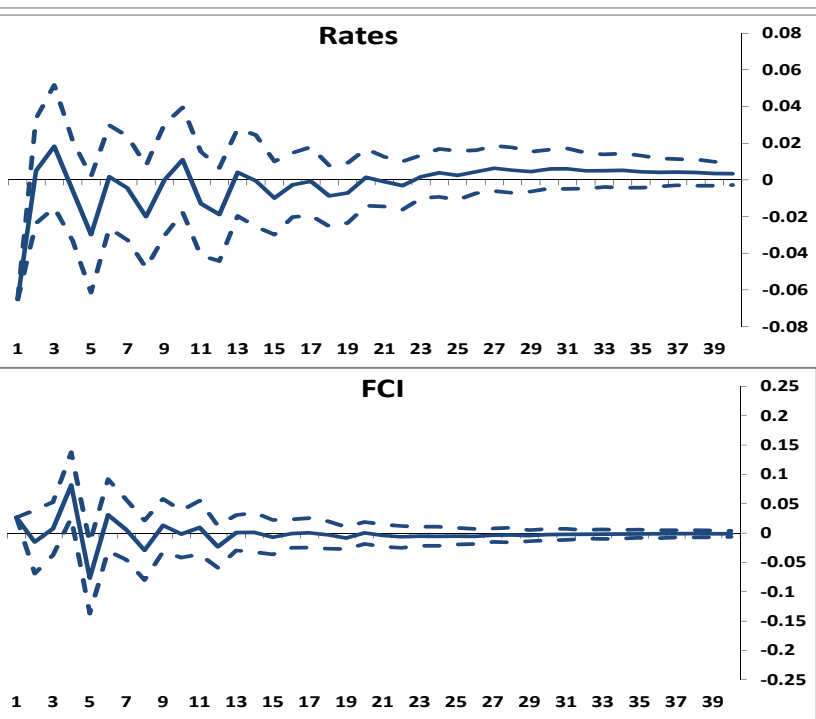

23

Figure 7: Impulse responses and 90\% confidence bounds following adverse monetary and credit 1 SD shocks, including FCI-M6. 


\section{Conclusion}

Financial Conditions Indices are popular devices for summarising the state of financial (credit) markets, and for forecasting in real time. In principle, although not always in practice, they differ from the related monetary conditions indices by being composed of real variables (e.g. spreads, rather than e.g. the levels of interest rates). Yet there is no consensus about how best to create them. In this paper we draw lessons from the macroeconomic and data-rich forecasting literature, which emphasises that there are likely to be a small number of common shocks or factors driving all sectors of the economy. So instead of combining (for example by principal components) variables described as 'financial' and calling the resulting index an FCI, we use the information in a large set of macroeconomic variables via MPLS to create a financial factor, which thus has a quasi-structural interpretation. Our new alternative specifically combines a large number of macroeconomic variables weighted by the joint covariance with subsets of financial indicators. Thus unlike standard FCIs which weight specific financial variables together by some means, our approach aims to weight latent factors from the macroeconomic data set using information from financial variables.

We compare this with the more common approach using a principal component of a medium sized set of relevant financial (and, following the literature, some monetary) indicators. Despite the contrasting methodology, our new FCI yields impressions of financial market tightness which are similar but differ in detail to those generated by the more standard principal components method. Both the standard and MPLS methods are useful for forecasting monthly GDP in a quasi-real-time recursive evaluation performed over three years to 2014, but the MPLS FCIs are superior to those based on the $\mathrm{PC}$ on average, and significantly so in more cases than the PC versions are superior to the MPLS. Using only the first factor, the MPLS-based FCIM6 emphasising private sector spreads is the best overall performer in a forecast race, and is particularly good at low forecast horizons, and outstandingly good for one month ahead. The second and third factors, which are less easily interpretable and which consequently we do not describe as FCIs, also improve forecast performance in the MPLS cases. The performance advantage of the (first factor) MPLS FCI over the PC version is largely maintained using different rolling windows, rather than a full available sample recursive analysis.

We also use the new FCI to identify credit supply shocks in an SVAR, where the main effects relative to one excluding FCI are to increase the positive impact of a credit shock on inflation, make lending more negative and spreads much higher.

Thus it seems that our new FCI does contain useful information about the current financial state and that the MPLS method of extracting factors from a large macro data set focussing on financial variables is useful for forecasting output growth.

Finally, we note some avenues that could be explored in future research. One would be map the contribution from the macroeconomic data in the $x$ set of variables on to the estimated 
FCI, possibly using the weights from rolling windows reported in Table 6 . This goes somewhat against the spirit of the idea that all macroeconomic data are driven by a small set of shocks, but might give users such as policymakers more insight into the mechanics of the FCI. The other is to do with estimation. The PC method estimates $406\left(\left(28^{2}-28\right) / 2\right)$ parameters while the PLS is somewhat more parsimonious ( 5 weights in the $y$ and then 82 weights on the $x$ ). But as discussed in Chun and Keles (2010) a sparse methodology offers a solution to a large parameter space. We note that extending such methods to our case would be a major exercise and therefore defer it to another paper. 


\section{A Appendix: macroeconomic data}

The two tables below set out the macroeconomic data (the $x$ ) used to construct the MPLS FCI. 


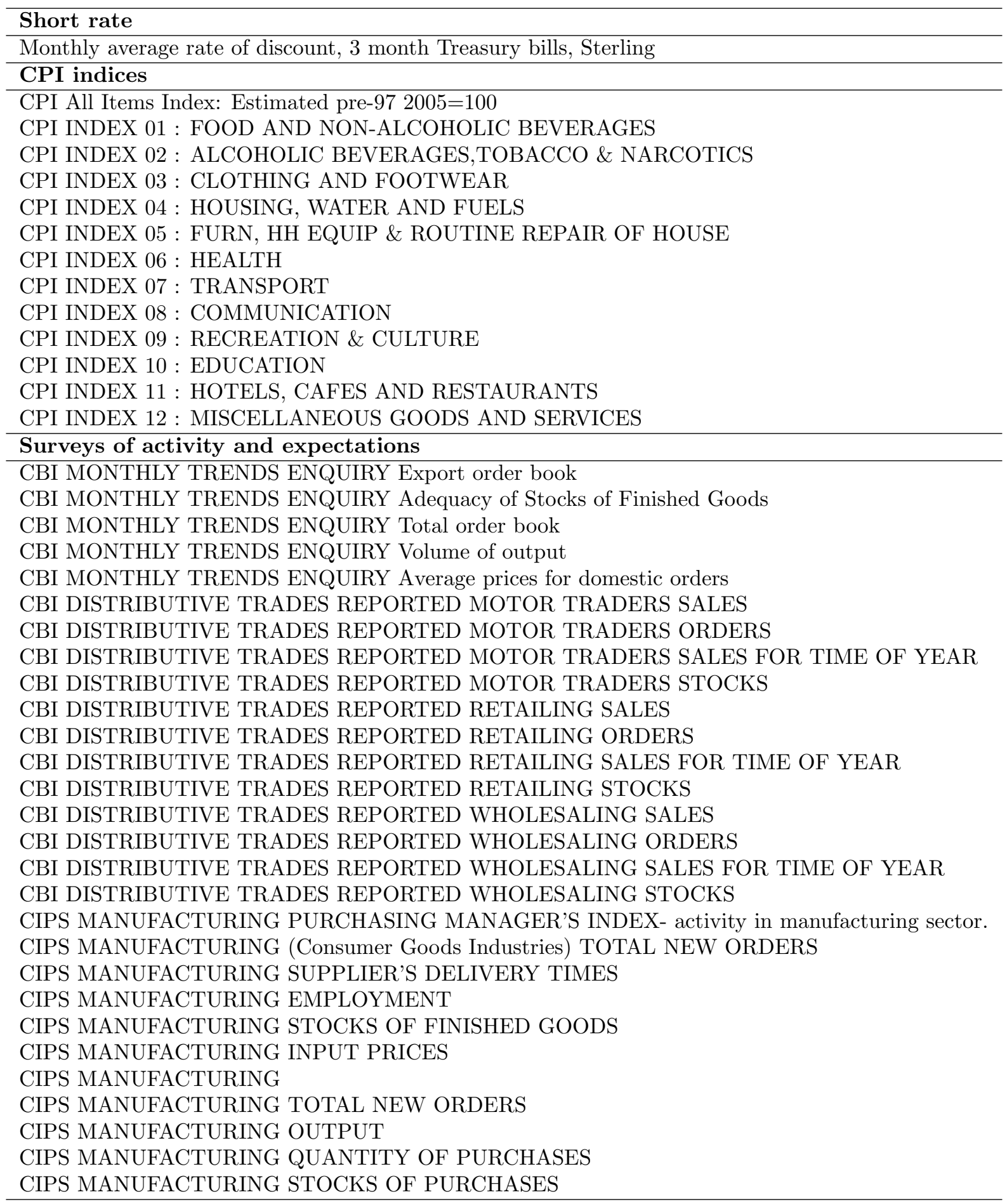

CPI: consumer price index; CBI: Confederation of British Industry; CIPS Chartered Institute of Purchasing and Supply survey

Sample 2004m01 to 2014m06; all variables transformed to stationarity

Table A: Macroeconomic data 


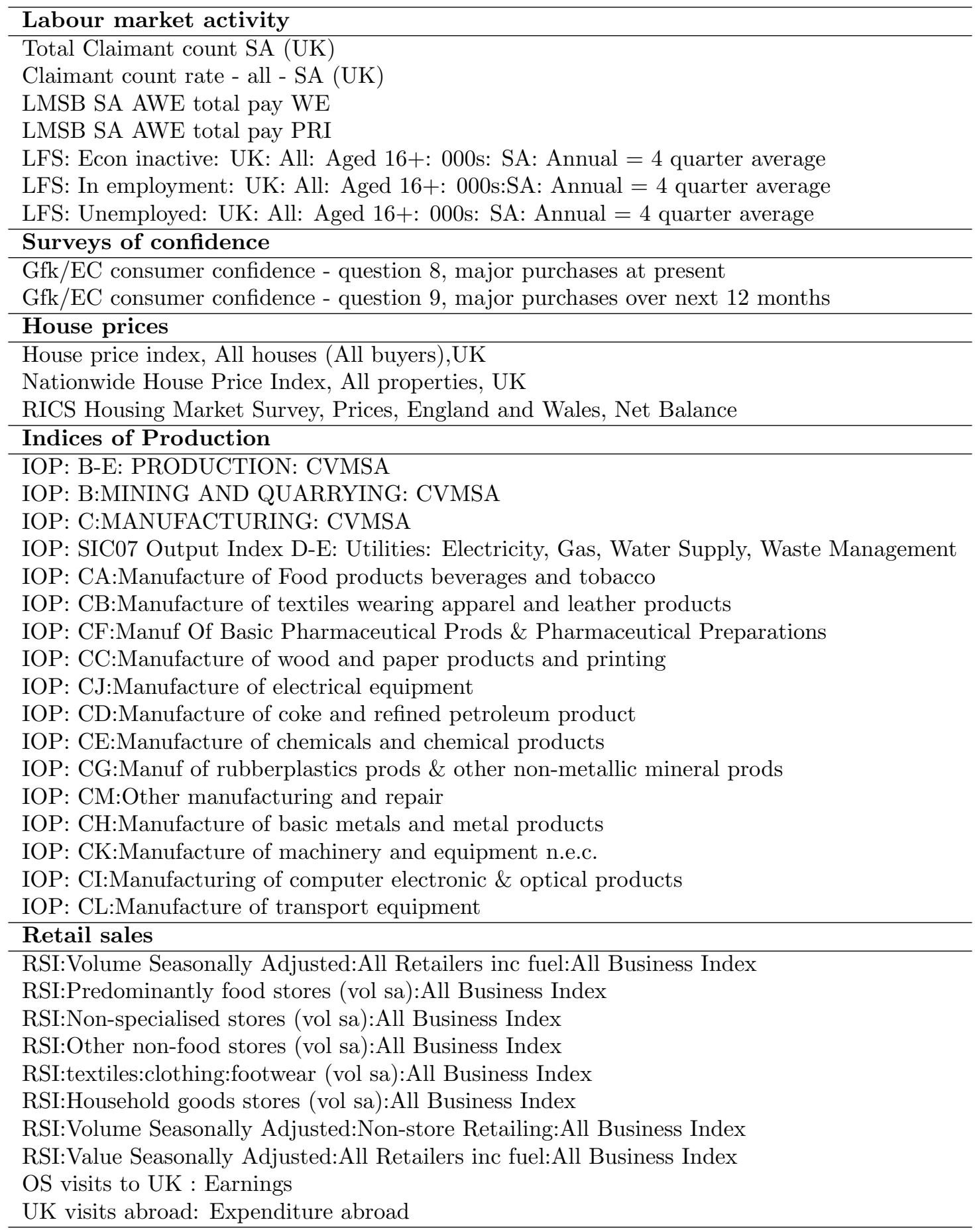

IOP index of industrial production; RSI retail sales index

Sample 2004m01 to 2014m06; all variables transformed to stationarity

Table B: Macroeconomic data (continued) 


\section{Acknowledgements}

We are grateful for comments and suggestions from three anonymous referees. 


\section{References}

Andrés, J., D. J. López-SAlido, And E. Nelson (2004): "Tobin's imperfect substitution in optimising general equilibrium," Journal of Money, Credit and Banking, 36, 665-90.

Angelopoulou, E., H. Balfoussia, and H. Gibson (2013): "Building a financial conditions index for the euro area and selected euro area countries: what does it tell us about the crisis?" ECB WP no. 1541 .

BAI, J. AND S. NG (2002): "Determining the number of factors in approximate factor models," Econometrica, 70, 191-221.

Barnett, A. And R. Thomas (2013): "Has weak lending and activity in the United Kingdom been driven by credit supply shocks?" Bank of England Working Paper No. 482.

Bates, B. J., M. Plagborg-Møller, J. H. Stock, and M. W. Watson (2013): "Consistent factor estimation in dynamic factor models with structural instability," Journal of Econometrics, 177, 289 - 304.

Busch, U., M. Scharnagl, and J. Scheithauer (2010): "Loan supply in Germany during the financial crisis," Discussion Paper Series 1: Economic Studies 2010:05, Deutsche Bundesbank.

Chun, H. And S. Keles (2010): "Sparse partial least squares regression for simultaneous dimension reduction and variable selection," Journal of the Royal Statistical Society B, 72, $3-25$.

Curdia, V. ANd M. WoOdFord (2011): "The central-bank balance sheet as an instrument of monetary policy," Journal of Monetary Economics, 58.

DE JONG, S. (1993): "SIMPLS: an alternative approach to partial least squares regression," Chemometrics and Intelligent Laboratory Systems, 18, 251-63.

Ericsson, N., E. Jansen, N. Kerbeshian, and R. Nymoen (1998): "Interpreting a monetary conditions index in economic policy," in Topics in Monetary Policy Modelling, Conference Papers Vol. 6, Bank for International Settlements.

Fernandez-Corugedo, E., M. McMahon, S. Millard, and L. Rachel (2011): "Understanding the macroeconomic effects of working capital in the United Kingdom," Bank of England Working Paper.

Gambetti, L. And A. Musso (2012): "Loan supply shocks and the business cycle," Working Paper Series, No. 1469, European Central Bank. 
Gauthier, C., C. Graham, And Y. Lui (2004): "Financial conditions indexes for Canada," $B o C W P$.

Gertler, M. And N. Kiyotaki (2010): "Financial intermediation and credit policy in business cycle analysis," .

Gilchrist, S., V. Yankov, And E. Zakrajsek (2009): "Credit market shocks and economic fluctuations: evidence from corporate bond and stock markets," Journal of Monetary Economics, 56, 471-93.

Gilchrist, S. And E. ZakrajSek (2012): "Credit spreads and business cycle fluctuations," American Economic Review, 102, 1692-720.

Giraitis, L., G. Kapetanios, and S. Price (2013a): "Adaptive forecasting in the presence of recent and ongoing structural change," Forthcoming in the Journal of Econometrics.

Giraitis, L., G. Kapetanios, and T. Yates (2013b): "Inference on multivariate stochastic time varying coefficient models," QMUL unpublished.

Groen, J. J., G. Kapetanios, and S. Price (2009): "A real time evaluation of Bank of England forecasts of inflation and growth," International Journal of Forecasting, 25, 74-80.

Groen, J. J. J. and G. Kapetanios (2013): "Model Selection Criteria for Factor-Augmented Regressions," Oxford Bulletin of Economics and Statistics, 75, 37-63.

Guichard, S., D. Haugh, and D. Turner (2009): "Quantifying the effect of financial conditions in the euro area, Japan, United Kingdom and the United States," OECD Economics Working Papers No. $67 \%$.

HARRISON, R. (2012): "Asset purchase policy at the effective lower bound for interest rates," Bank of England Working Paper.

Hatzius, J., P. Hooper, F. Mishkin, K. Schoenholtz, and M. Watson (2010): "Financial Conditions Indexes: a fresh look after the financial crisis," Working Paper.

Helbling, T., R. Huidron, M. A. Kose, and C. Otrok (2011): "Do credit shocks matter? A global perspecive," European Economic Review, 55, 340-53.

Helland, I. S. (1988): "On the structure of Partial Least Squares Regression," Communications in Statistics - Simulation and Computation, 17, 581-607.

(1990): "Partial Least Squares Regression and statistical models," Scandinavian Journal of Statistics, 17, 97-114.

Koop, G. And D. Korobilis (2013): "A new index of Financial Conditions," Working Paper. 
Millard, S. ANd A. Nicolae (2014): "The effect of the financial crisis on TFP growth: a general equilibrium approach," Bank of England Working Paper.

Mueller, P. (2009): "Credit spreads and real activity," EFA 2008 Athens Meetings Paper.

Paries, M. D., L. Maurin, And D. Moccero (2014): "Financial conditions index and credit supply shocks for the euro area," ECB Working Paper Series.

Pearson, K. (1901): "On lines and planes of closest fit to systems of points in space," Philosophical Magazine, 2, 559-572.

Peersman, G. (2012): "Bank lending shocks and the euro area business cycle," Ghent University WP.

Rossi, B. (2013): "Advances in Forecasting under Instability," in Handbook of Economic Forecasting, ed. by G. Elliott and A. Timmermann, 1203-1324.

Stock, J. H. AND M. W. WAtson (2002): "Forecasting using principal components from a large number of predictors," Journal of the American Statistical Association, 97, 1167-1179. (2009): "Forecasting in dynamic factor models subject to structural instability," in The Methodology and Practice of Econometrics: A Festschrift in Honour of David F. Hendry, ed. by D. F. Hendry, J. Castle, and N. Shephard, Oxford University Press, 173 - 205.

Swiston, A. (2010): "A U.S. financial conditions index: putting credit where credit is due," IMF WP/08/161.

Wold, H. (1982): "Soft modeling. The basic design and some extensions," in Systems Under Indirect Observation, Vol. 2, ed. by K.-G. Jöreskog and H. Wold, Amsterdam: North-Holland. 